DOI: $10.3901 / J M E .2019 .13 .038$

\title{
基于声模态的压气机/风扇气路故障诊断*
}

\author{
程 礼 $^{1,2}$ 杨武奎 ${ }^{1,3}$ 梁 涛 $^{1}$ 文 璧 $^{4}$ 姚东野 ${ }^{1}$ \\ (1. 空军工程大学航空工程学院 西安 710038; \\ 2. 先进航空发动机协同创新中心 北京 100191 ; \\ 3. 航泰动力机器厂 襄阳 441002; \\ 4. 中国航发浴轮研究院航空发动机高空模拟技术重点实验室 绵阳 621700)
}

\begin{abstract}
摘要: 通过压气机/风扇流道中声模态产生机理分析, 阐述了压气机/风扇效率与声模态之间的联系, 探索基于声模态的航空 发动机气路故障的诊断方法。基于奇异值分解理论, 提出了一种声模态信号的提取方法, 并通过某风扇试验器试验数据处理, 成功绘制出了声模态时序图。根据声模态时序图的波峰、波谷分布, 辨识出该风扇在亚音速工作范围内既有前行声模态, 又 有后行声模态。而当后行声模态居于主导地位时, 风扇效率明显下降, 偏离设计指标, 与实际故障现象相吻合, 验证了声模 态诊断方法的可行性。
\end{abstract}

关键词: 航空发动机; 压气机/风扇; 故障诊断; 声模态; 奇异值分解

中图分类号: TG156

\section{Failure Diagnosis on Gas Path of Fan/compressor Based on Acoustic Mode}

\author{
CHENG Li $^{1,2}$ YANG Wukui ${ }^{1,3}$ LIANG Tao ${ }^{1}$ WEN Bi $^{4}$ YAO Dongye ${ }^{1}$ \\ (1. Aeronautics Engineering College, Air Force Engineering University, Xi'an 710038; \\ (2. Collaborative Innovation Center for Advanced Engine, Beijing 100191; \\ 3. Hangtai Power Machinery Factory, Xiangyang 441002; \\ 4. Key Lab. of Engine Altitude Simulation Technology, Gas Turbine Establishment of \\ Aero Engine Corporation of China, Mianyang 621700)
}

\begin{abstract}
The relation between efficiency of compressor/fan and acoustic mode is set forth by analysing generating mechanism of acoustic mode in the flow duct of fan/compressor, the failure diagnosis method of aero-engine's gas path based on acoustic mode is explored. On the basis of singular value decomposition(SVD) theory,a kind of taking-up method for acoustic mode sensor is put forward,and in accordance with test data processing of a type of fan tester,time sequence diagram of acoustic mode is successfully drawn, both forward acoustic mode and backward acoustic mode co-existence are distinguished during the subsonic work range of the fan by wave crest and valley distribution of acoustic mode's time sequence diagram, at the same time the fan efficiency obviously decreasing is found when backward acoustic mode is in the leading status, and design requirement is deviated, the calculation results are in good agreement with the practical failure mode, the diagnosis method of acoustic mode is feasible by verifying.
\end{abstract}

Key words: aero-engine; compressorfan; failure diagnosis; acoustic mode; singular value decomposition(SVD)

\section{0 前言}

航空发动机是飞机的动力装置, 其稳定可靠地 工作是飞机完成预定任务、保证飞机安全的基本保 证。目前, 状态监控与故障诊断技术已经广泛用于 航空发动机的气路、整机振动、滑油系统等 ${ }^{[1]}$ 。其

* 国家重点基础研究发展计划资助项目(973 计划, 2015CB057400)。 20180720 收到初稿, 20181123 收到修改稿
中，气路诊断涉及压气机/风扇、燃烧室、涡轮、加 力燃烧室和喷管等部件, 由于实际流场状况十分复 杂, 参数测量比较困难, 诊断效果不很理想。

关于航空发动机气路噪声产生机理与降噪方面 的研究始于 20 世纪中叶。TYLER 等 ${ }^{[2]}$ 最早系统研 究了声模态产生的机理和影响因素, 为风扇和压气 机噪声研究采用声模态奠定了理论基础。JOPPA ${ }^{[3-4]}$ 等通过研究发现, 掌握了压气机等旋转机械噪声的 产生机制、声源位置、传播方式对于设计低噪声航 空发动机十分重要, 而声模态测量是识别旋转机 
械噪声源物理机制、分辨主要噪声源位置以及分析 噪声传播方式的一个重要方法。王良锋等 ${ }^{[5]}$ 利用轴 流风扇管道内部周向均匀分布的麦克风阵列, 对风 扇在高背景噪声和较大硬壁反射条件下的管道周向 声模态进行了试验测量, 利用 CC 和 RMS 模态分解 技术得到了主要的周向声模态振幅。梁东等 ${ }^{[6]}$ 通过 研究, 发展了模态测量的数据分析方法, 并提出了 非均布模态测量结果的分析方法, 解决了测点非均 布及测点数目非 2 的整数次倍的问题。

近年来, 在航空发动机压气机工作中发现了导 致叶片疲劳断裂的声共振现象, 其驱动源多为气动 激励, 其物理本质是气体诱发腔体的声学共振问 题 ${ }^{[7]}$ 。BERND 等 ${ }^{[7]}$ 通过简化的螺旋式声模态模型构 建, 解释了高速轴流式压气机声共振产生的原因。 $\mathrm{CAMP}^{[8]}$ 在压气机上通过试验观测出多级压气机的 声共振现象, 并通过理论分析判断出声共振现象。 杨明绥等 ${ }^{[9]}$ 通过对某型压气机进行试验研究, 发现 该压气机的故障特点与国外开展的声共振研究结果 相似。

综上研究工作的核心内容都与声模态和声共振 有关。既然气动噪声是发动机工作过程中流道内外 气体流动、燃烧的直接结果, 其中也必然携带有大 量关于发动机性能状态的信息, 基于声模态的故障 诊断技术是一个新的研究方向。国内外在这方面均 鲜有报道。

本文以航空发动机压气机/风扇为对象, 探索基 于声模态提取进行气路故障诊断的方法。首先, 通 过压气机/风扇流道中声模态产生机理分析, 阐述了 压气机/风扇效率与声模态之间的联系; 其次, 在奇 异值分解基础上提出声模态提取方法, 并完成某风 扇试验器试验数据处理; 最后, 基于该风扇试验器 在亚音速工作范围的声模态分析, 验证诊断方法的 可行性。

\section{1 压气机/风扇内流声模态形成机理 与气路故障}

\section{1 声模态及其形成机理}

航空发动机气路可以简化为管道内流流场。基 于圆管、连续性、均匀流和无粘假设, 并结合动量 守恒方程和连续方程, 可得其中声传播理论分析模 型, 即对流波动方程

$$
\nabla^{2} p-\frac{1}{c_{0}^{2}} \frac{D^{2} p}{D t^{2}}=0
$$

式中, $c_{0}$ 为声速; 柱坐标系中 $\nabla^{2}$ 的算式为

$$
\nabla^{2}=\frac{\partial^{2}}{\partial r^{2}}+\frac{1}{r} \frac{\partial}{\partial r}+\frac{1}{r^{2}} \frac{\partial}{\partial \theta^{2}}+\frac{\partial^{2}}{\partial z^{2}}
$$

根据微分计算, 式(1)得到一个特解

$$
P_{m, n}(r, \theta, z, t)=
$$

$A_{m, n} J_{m}\left(K_{r}(m, n) \bullet r\right) \exp \left[i\left(m \theta+\varphi_{m}\right)\right] \exp \left(k_{s} z-\omega t\right)$

对于每一数值组合 $(m, n)$, 式(2)都有一个对 应的解, 而这个解是方程式(1)的一个特解。方程 式(1)的解应是所有简谐波, 其中包括 $(0,0)$ 阶平面 波及其余 $(m, n)$ 阶高阶波的叠加, 因此管中总声压 可表示为

$$
\begin{gathered}
P_{m, n}(r, \theta, z, t)=\sum_{m=0}^{\infty} \sum_{n=0}^{\infty} P_{m, n}(r, \theta, z, t)= \\
\sum_{m=0}^{\infty} \sum_{n=0}^{\infty} A_{m, n} J_{m}\left(K_{r}(m, n) \cdot r\right) \exp \left[i\left(m \theta+\varphi_{m}\right)\right] \\
\exp \left(k_{s} z-\omega t\right)
\end{gathered}
$$

由上式可知, 某频率分量是由周向模态和径向 模态叠加而成。本文只考虑周向模态, $A(m, n)$ 表示 目标频率为 $\omega$ 、周向模态为 $m$ 阶处的声压幅值。 $A(m, \omega)$ 是一个只与 $m$ 和 $\omega$ 有关的量

$$
P(\omega, \theta)=\sum_{m=-\infty}^{+\infty} A(m, n) \exp (i m \theta)(m=0, \pm 1, \pm 2, \cdots)
$$

由于压气机/风扇是对气流做功的部件, 应先考 虑转子自身的情况, 再考虑转子与静子之间干涉所 引起的噪声源, 通常在这两种情况下产生的旋转压 力模态就是声模态。实际上式(4)包含了顺流向传播 和逆流向传播的两组声模态, 分别称为前行声模态 和后行声模态。

在压气机/风扇中, 稳定的叶片气动载荷和叶片 的厚度影响较大, 而由于湍流和叶片振动导致涡流 散布、瞬间的气动载荷变化影响较小。气动和厚度 影响的压力场可以通过转子叶片的任意一半径上的 压力线表现出来。如果转子上有 $B$ 个叶片, 那么沿 着转子周向就可均匀分为 $B$ 个 $360 / B$ 度的间隔, 相 关的压力线在这个间隔内被重复。同时压力分布在 叶片前面一个固定位置上可以作为一个角度位置函 数被表现出来。压力分布取决于叶片上的压力线, 并遵循傅里叶级数的周期函数, 在任意半径和周向 位置的压力可用角度和时间的函数表示

$$
p(\theta, t)=\sum_{n=o}^{\infty} a_{n} \cos \left[n B(\theta-\Omega t)+\varphi_{n}\right]
$$

式中, $\Omega=2 n \pi$ 为转子的角速度; $a_{n}$ 为振幅, $\varphi_{n}$ 为 相位。 
为保证效率不衰减, 压气机/风扇的转子与静子 之间的间隙不能过大, 为了避免碰摩, 转子与静子之 间的间隙又不能过小。工作过程中, 转子与静子之间 产生一定频率的振动模态, 如果干涉模式的波瓣少于 叶片的数量, 必须比转子转动的更快才能达到相同的 频率, 因此当转子顶端速度是亚音速时, 通过干涉可 以产生传播模态, 周期性压力脉动仍可以通过傅里叶 公式表述。除此外, 模态数量与转子、静子之间的关 系满足 $m=n B+k V(k=\cdots,-1,0,2, \cdots)$, 其中 $B$ 为转子叶片数量, $V$ 为静子叶片数量 ${ }^{[2]}$ 。

\section{2 相关气路故障}

\subsection{1 声共振导致叶片疲劳断裂故障}

压气机空腔既然是气体流动的通道, 在运转过 程中也是受流体和声介质波动影响最严重的部位。 在空腔中, 作为激励源的叶片会在运转过程中产生 叶片通过频率，如果其频率与空腔声模态的固有频 率相近, 就会引起空腔声模态的共振。从而强化对 叶片气体激振，造成冲击，使之疲劳破坏。

\subsection{2 后行声模态导致压气机/风扇效率下降故障}

在压气机气路通道中, 声模态是叠加在气流中的 扰动, 扰动能量可大可小, 声共振就属于强扰动。前 行声模态比较容易理解, 由于其与气流流动方向相 同, 会引起叶片的振动。后行声模态的情况比较复杂, 由于其与气流流动方向相反, 其不但会引起叶片振 动, 而且会对气流形成堵塞作用, 如果此时扰动能量 又较强, 就可能导致压气机/风扇的效率下降。

\section{2 基于奇异值分解的声模态提取方法}

目前, 有关声模态研究都以文献[2]为基础, 只 绘制出了声模态的形状图, 不能绘制出声模态的时 序图, 主要原因在于对信号处理时忽略了实时相位 特征。

基于 Hankel 矩阵奇异值分解的一个非常有用 的特性: 线性分解特性, 分解不但保持幅值, 而且 保持相位不变 ${ }^{[10-11]}$ 。另外, 各奇异值重构信号仅包 含单一频率成分, 具有很好的窄带滤波特性。除上 述两点内容外, 奇异值分解技术还具有很好的降噪 效果, 可以在强噪声背景环境下准确提取设备的故 障特征信号, 提高信噪比 ${ }^{[12]}$ 。

本文在奇异值分解基础上构建声模态的提取方 法, 对某风扇试验器试验数据进行处理, 提取了该 风扇在亚音速工作范围的声模态, 验证了方法的可 行性。

\section{1 基本步骤}

基于奇异值分解的声模态提取方法充分利用奇异
值分解的基本特性和原理，主要包括以下四个步骤。

(1) 根据实际需要并考虑计算时间, 对原始信 号进行截取, 然后对所截取的信号进行频谱分析, 根据其频谱图并再结合风扇转速确定目标信号频率 及其幅值大小顺序。

(2) 将截取的信号按要求构建成一定列数(行数) 的 Hankel 矩阵, 文献[12]中表明, 当 Hankel 矩阵结 构当列数满足: $q=N / 2$ 或 $q=(N+1) / 2$ 时, 此时 提取效果最佳, 具体由信号长度的奇、偶性确定, 同时 $p=N-q+1$ 。

（3）利用构建好的 Hankel 矩阵进行奇异值分 解, 并用步骤(1)得到的基本信息, 将目标信号与奇 异值对应起来，得到目标奇异值。

（4）利用目标奇异值进行重构, 提取得到目标信 号，最后对提取得到的信号再进行频谱分析，将幅 值和频率与原始信号比对, 确保提取结果准确无误。

\section{2 奇异值分解}

截取的信号序列为 $\boldsymbol{X}=[x(1), x(2), \cdots, x(N)], N$ 为信号长度，则由其构建的 Hankel 矩阵为

$$
\boldsymbol{H}=\left[\begin{array}{cccc}
x(1) & x(2) & \ldots & x(q) \\
x(2) & x(3) & \ldots & x(q+1) \\
M & M & M & M \\
x(p) & x(p+1) & L & x(N)
\end{array}\right]_{p \times q}
$$

式中 $1<q<N, p=N-q+1$, 不失一般性取 $p \leqslant q$ 。 对 $H$ 矩阵进行 SVD 可得

$$
\boldsymbol{H}=\boldsymbol{U}\left[\begin{array}{cccc}
\sigma_{1} & & & \\
& \sigma_{2} & & \\
& & \ddots & \\
& & & \sigma_{q}
\end{array}\right]_{p \times q} \boldsymbol{V}^{\mathrm{T}}
$$

其中 $\sigma$ 为 Hankel 矩阵奇异值, 满足 $\sigma_{1} \geqslant \sigma_{2} \geqslant \cdots \geqslant \sigma_{q}$ 。 将式(7)改写为用左右奇异矢量 $\boldsymbol{u}_{i}$ 和 $\boldsymbol{v}_{i}$ 表示的形式, 则

$$
\boldsymbol{H}=\sigma_{1} \boldsymbol{u}_{1} \boldsymbol{v}_{1}{ }^{\mathrm{T}}+\sigma_{2} \boldsymbol{u}_{2} \boldsymbol{v}_{2}{ }^{\mathrm{T}}+\cdots+\sigma_{q} \boldsymbol{u}_{q} \boldsymbol{v}_{q}^{\mathrm{T}}
$$

式中, $\boldsymbol{u}_{i} \in \boldsymbol{R}^{p \times 1}, v_{i} \in \boldsymbol{R}^{q \times 1}, i=1,2, \cdots, p$ 。令 $\boldsymbol{H}_{i}=\sigma_{i} \boldsymbol{u}_{i} \boldsymbol{v}_{i}^{\mathrm{T}}$, 则 $\boldsymbol{H}_{i} \in \boldsymbol{R}^{p \times q}$ 。目标分量对应奇异值为 $\sigma_{1}$, 因此目 标信号矩阵取 $\boldsymbol{H}_{1}=\sigma_{1} \boldsymbol{u}_{1} \boldsymbol{v}_{1}{ }^{\mathrm{T}}$ 。采用平均修正思路, 对 $\boldsymbol{H}_{1}$ 矩阵中每一反对角线元素取均值, 则重构分量信 号可以表示为

$$
x_{1}(k)=\frac{1}{n-m+1} \sum_{j=m}^{n} \boldsymbol{H}_{i}(k-j+1, j)
$$

式中, $m=\max (1, k-p+1) ; n=\min (q, k), k=1,2, \cdots, N$ 。 得到目标信号

$$
X=x_{1}+x_{2}+\cdots+x_{p}=\sum_{i=1}^{p} x_{i}
$$




\section{3 声模态提取试验与结果分析}

\section{1 试验装置}

某二级跨音速风扇试验器结构参数如表 1 所 示, 结构如图 1a 所示。转速超过 $11000 \mathrm{r} / \mathrm{min}$ 后, 该风扇叶尖处于超音速状态。

\section{表 1 风扇试验器结构参数}

\begin{tabular}{cc}
\hline 名称 & 叶片数目/片 \\
\hline 进气支板 & 18 \\
一级转子 & 17 \\
一级静子 & 42 \\
二级转子 & 31 \\
二级静子 & 86 \\
\hline
\end{tabular}

试验发现, 风扇转速在 9400 11 000 r/min 范围 内效率下降，没有达到设计指标，原因不明。

针对该故障现象，在风扇进气支板前约 $10 \mathrm{~mm}$ 的气流通道壁面上沿周向均匀布置 24 只传声器同 步采集声压信号(图 1), 风扇旋转方向为顺时针方 向, 采样频率为 $25000 \mathrm{~Hz}$, 探索基于声模态的风扇 故障诊断方法。

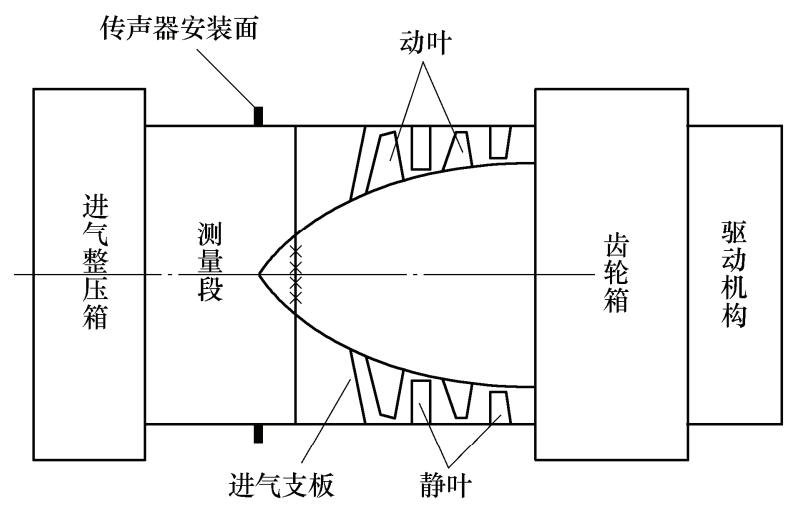

(a) 某二级跨音速风扇试验器结构示意图

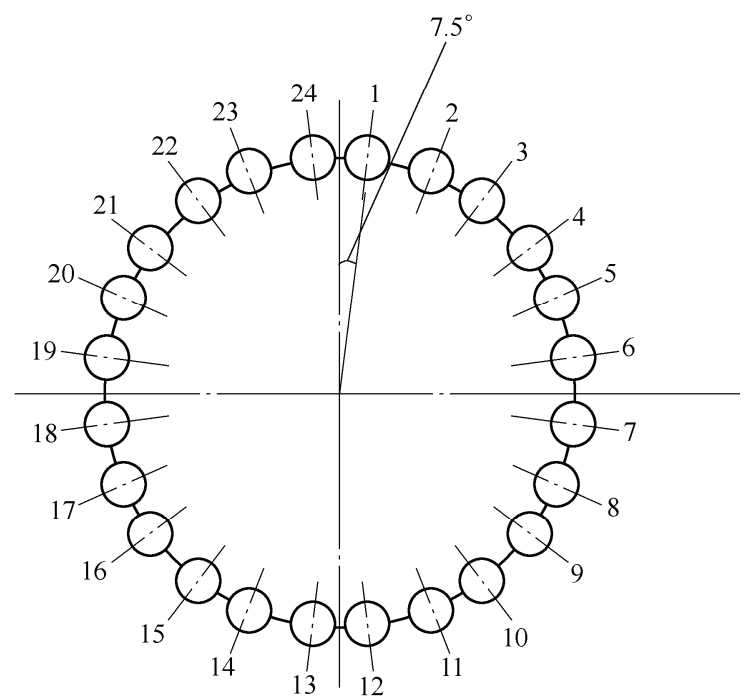

(b) 传声器位置分布图

图 1 传声器安装示意图
试验包括加速、稳定(超音速状态)和减速三个 阶段, 持续 $16.8 \mathrm{~min}$, 其声压时域波形如图 2 所示。 试验中, 2 号、16 号、24 号等 3 只传声器发生故障, 没有采到数据。

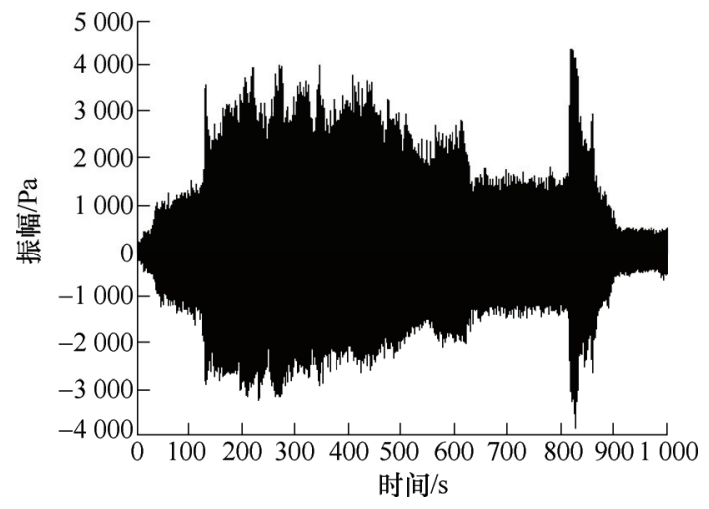

图 2 信号时域波形

由图 2 可知, 发现 $620 \mathrm{~s}$ 以前, 试验器处于加 速状态，且测信号幅值较大，便于进行声模态提取; 在 $620 \sim 820 \mathrm{~s}$ 之间, 试验器处于稳定阶段, 由于此 时叶尖为超音速状态，声音传播受到抑制，幅值较 小; 在 $820 \mathrm{~s}$ 之后, 试验器处于减速状态, 所测信 号幅值先是明显增加后减小, 也可进行声模态提取。

\section{2 试验数据预处理}

按第 2.1 节步骤截取满足提取声模态图要求的 数据段进行分析，一来保证期间风扇转速恒定，二 来保证计算速度较快。截取长度为 $1 \mathrm{~s}$ 的信号, 时 域与频谱分析结果如图 3a、3b 所示。

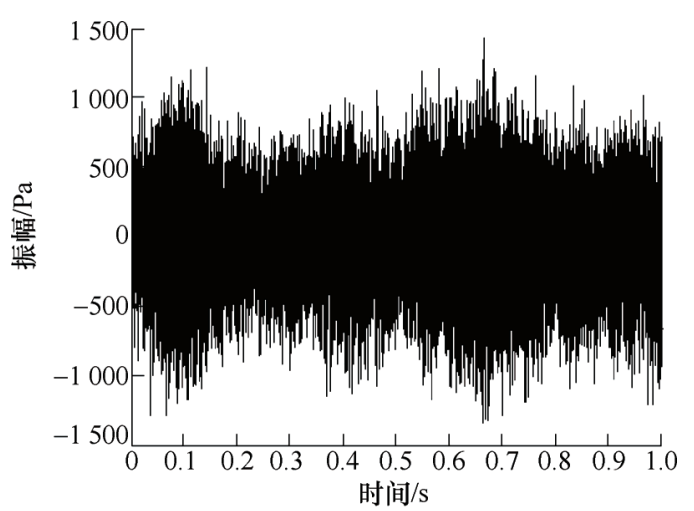

(a) 信号时域

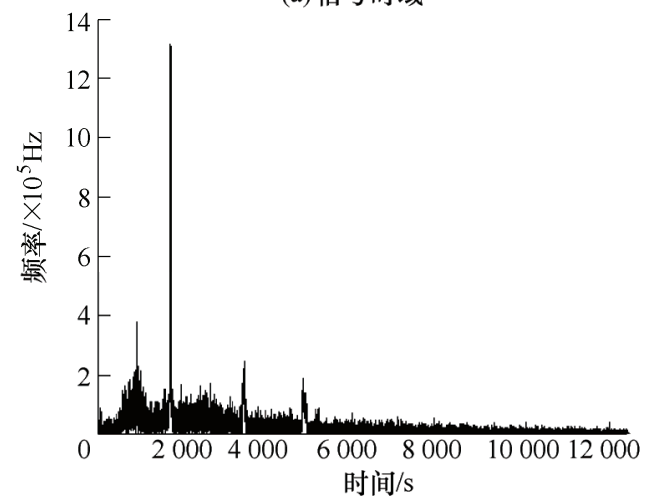

(b) 信号频谱

图 3 试验器声信号 
此时风扇转速约为 $6000 \mathrm{r} / \mathrm{min}$, 由于试验器转 子一级动叶为 17 片, 可以确定频谱图中 $1700 \mathrm{~Hz}$ 的信号即为叶尖通过频率信号, 也是下面将要提取 的目标信号。

按照第 2.2 节奇异值分解方法, 提取后的目标 频谱图(图 4), 对比可以看出与目标频率一致, 提取 结果有效。

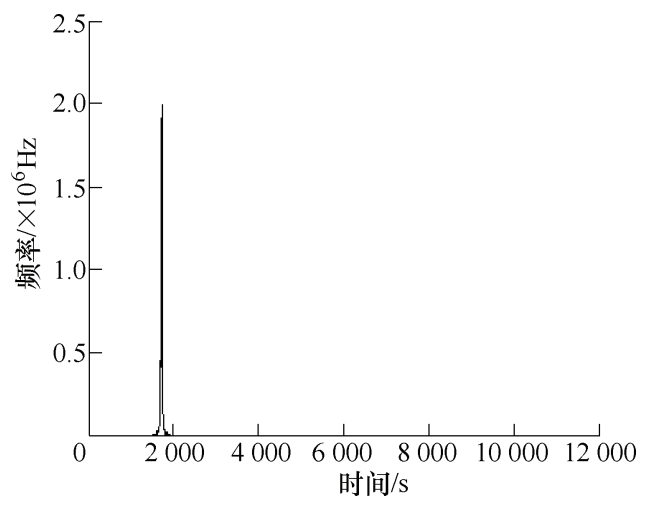

图 4 提取后目标分量频谱图

\section{3 声模态提取}

由于三只传声器测量时发生故障, 所得数据无 效, 在绘制声模态图时进行了摒弃。

利用奇异值分解方法对有效的 21 个通道的声 模态进行提取, 并将提取的数据绘制在一张图上, 其中横坐标代表每个传声器的位置, 纵坐标代表时 间, 用不同颜色(暖色为峰值、冷色为谷值)代表所 测得声压大小, 由此便得到声模态时序图。

首先, 分别绘制低(4000 r/min 左右)、中(7000 $\mathrm{r} / \mathrm{min}$ 左右)、高(10 $000 \mathrm{r} / \mathrm{min}$ 左右)三种转速状态 下, 加速段和减速段的声模态图。结果表明: 无 论加速段还是减速段, 在同一转速下, 声模态结 果都是基本一致的。图 5 和图 6 分别是中、低速 时的结果。由图可知, 此时, 声模态压力波波峰 和波谷沿左上方向右下方倾斜分布, 方向一致, 几乎平行。在每一个时间截面上, 声模态只有一 个波峰和一个波谷, 即 $m=1$ 。此时实测试验器效 率满足设计要求。

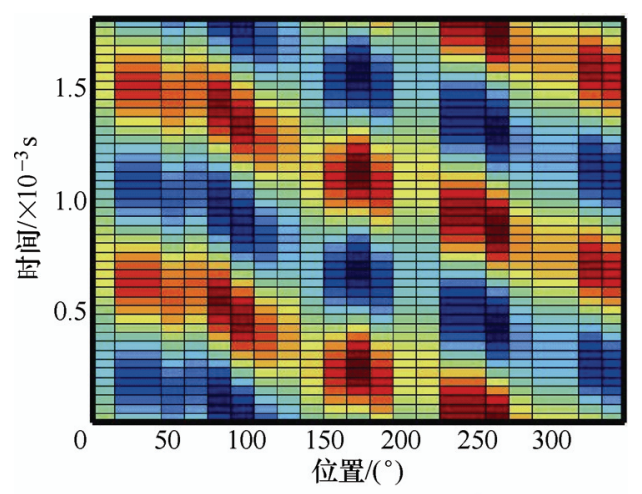

(a) $4044 \mathrm{r} / \mathrm{min}$ 转速加速段声模态

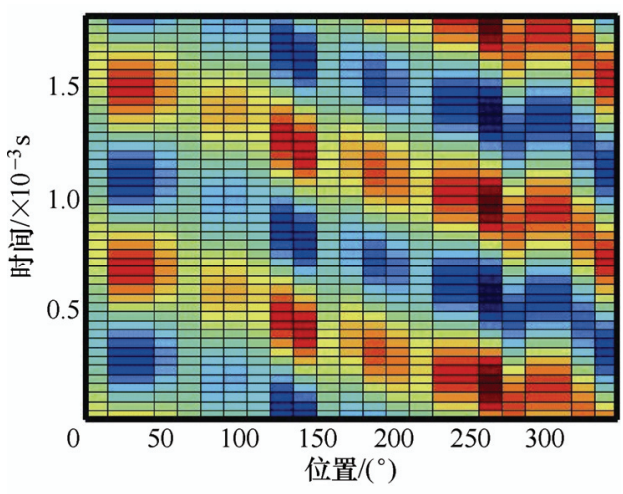

(b) $4411 \mathrm{r} / \mathrm{min}$ 转速减速段声模态

图 5 低速时的声模态图

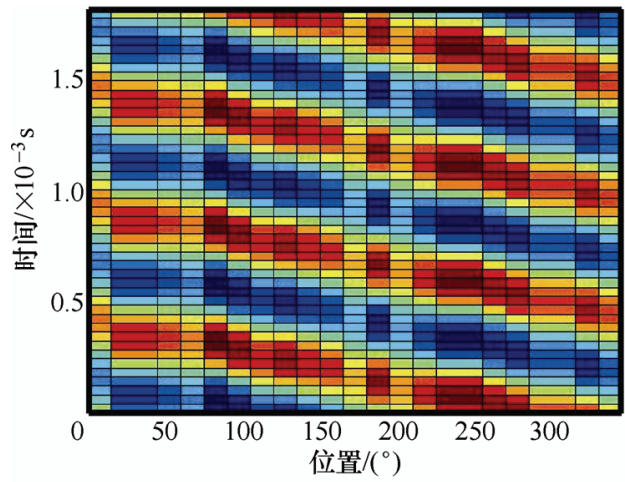

(a) $6840 \mathrm{r} / \mathrm{min}$ 转速加速段声模态

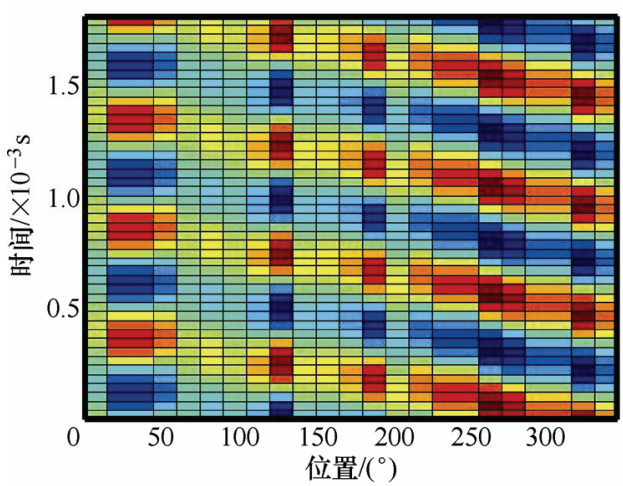

(b) $7281 \mathrm{r} / \mathrm{min}$ 转速减速段声模态

图 6 中速时的声模态图

图 7 给出的是高转速时的声模态图, 其与图 5、 图 6 具有较大差别。从图 7a 可以看出, 此时, 声模 态波峰和波谷倾斜方向不再平行, 波峰和波谷也没 有中、低速时那么明显, 而是出现混叠, 有换向趋 势。随着转速进一步增加, 由图 $7 \mathrm{~b}$ 可以看出, 此时, 声模态压力波波峰和波谷沿左下方向右上方倾斜分 布, 方向一致, 几乎平行, 但中低速时倾斜方向相 反。在每一个时间截面上，声模态有五个波峰和五 个波谷, 即 $m=5$ 。此时实测试验器效率不满足设计 要求。

\section{4 故障相关分析}

为了进一步对比分析, 在提取中的 21 组有效数 据中, 选取了 3 号、 4 号、 5 号、6 号、7 号等 5 只 连续分布的传声器的测量数据。分别绘制在相同时 
间段内提取的中、高速状态下对应声模态时序波形 图(图 8)。

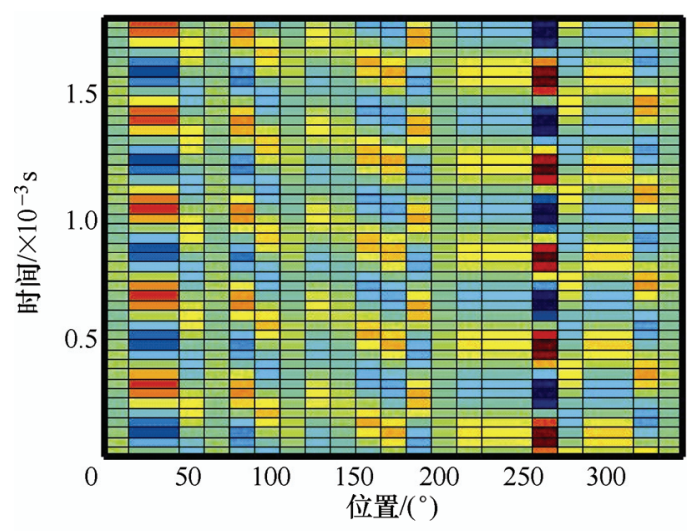

(a) $9557 \mathrm{r} / \mathrm{min}$ 转速声模态

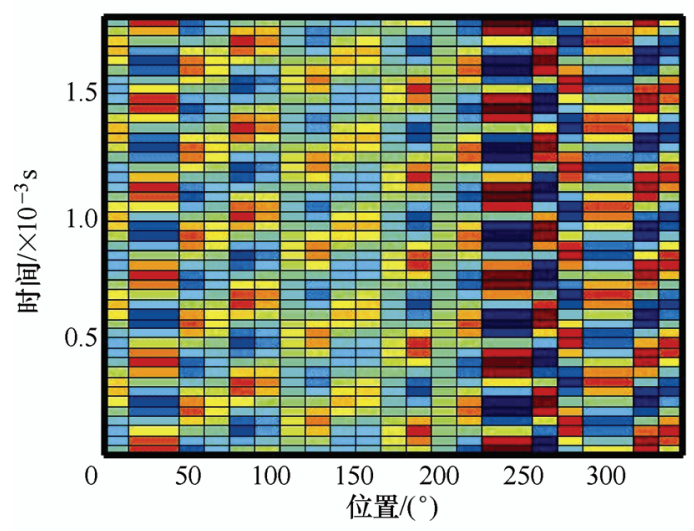

(b) $10072 \mathrm{r} / \mathrm{min}$ 转速声模态

图 7 高速时的声模态图
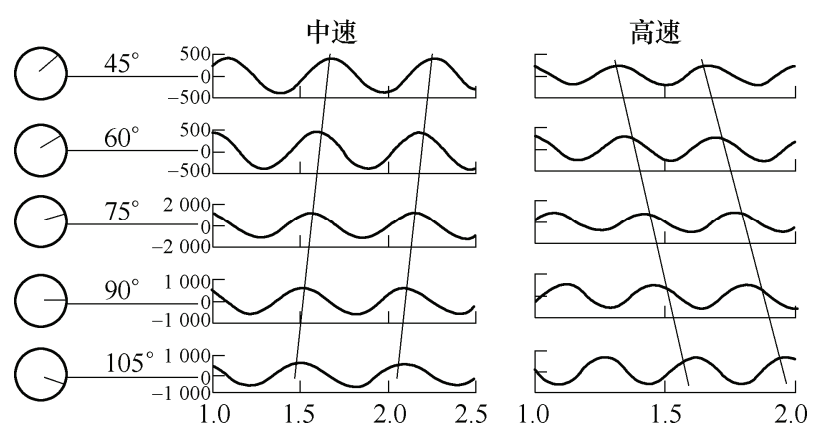

图 8 压力场与相位之间的关系

在中速(6 $024 \mathrm{r} / \mathrm{min})$ 状态时, 相邻两个传声器测 得声波时序图存在相位差, 沿试验器旋转方向, 后 一个传声器较前一个传声器落后约 $51.6^{\circ}$, 传播与 试验器气流方向一致, 据此可以判断此时声模态为 前行声模态。声扰动不会影响风扇效率。

在高速(10 $058 \mathrm{r} / \mathrm{min}$ ) 状态时, 相邻两个传声器 测得声波时序图同样存在相位差, 沿试验器旋转方 向, 后一个传声器较前一个传声器超前约 $40.1^{\circ}$, 传播与试验器气流方向相反, 据此可以判断此时声 模态为后行声模态。声扰动对气流起阻碍作用, 会
影响风扇效率。

虽然通过上述试验结果, 发现了后行声模态与 风扇效率下降之间有定性的对应关系, 但定量关系 还需进一步研究。

\section{4 结论}

论文通过压气机/风扇流道中声模态产生机理 分析, 阐述压气机/风扇效率与声模态之间的联系, 探索了基于声模态的航空发动机气路故障的诊断方 法。研究结果可得结论如下。

（1）基于奇异值分解理论构建的声模态提取方 法, 能够有效地保证特征信号的相位关系, 成功绘 出了声模态时序图。根据声模态时序图的波峰、波 谷分布, 可以比较容易地辨识出前行声模态和后行 声模态。

(2) 通过某风扇试验器声压数据处理, 辨识出 该风扇在亚音速工作范围内既有前行声模态, 又有 后行声模态。当前行波居于主导地位时, 风扇效率 较高, 满足设计指标; 而当后行波居于主导地位时, 风扇效率明显下降, 偏离设计指标, 与实际故障现 象相吻合，验证了声模态诊断方法的可行性。

\section{参 考 文 献}

[1] 陈卫, 程礼, 李全通, 等. 航空发动机监控技术 [M]. 北 京: 国防工业出版社, 2011.

CHEN Wei, CHENG Li, LI Quantong, et al. Aero engine monitoring technology[M]. Beijing: National Defence Industry Press, 2011.

[2] TYLER J M, SOFRIN T G. Axial flow compressor noise studies[J]. Transactions of the Society of Automotive Engineers, 1962, 70: 309-332.

[3] JOPPA P D. Acoustic mode measurements in a inlet of a turbofan engine[J]. Journal of Aircraft, 1987, 24(8): 557-593.

[4] MEYER H D, ENVIA E. Aeroacoustic analysis of turbofan noise generation[R]. NASA CR-4715, 1996.

[5] 王良锋, 乔渭阳, 纪良, 等. 轴流风扇/压气机管道周 向声模态的测量 [J].航空动力学报, 2014, 29(4): 917-926.

WANG Liangfeng, QIAO Weiyang, JI Liang, et al. In-duct circumferential acoustic mode measurement of axial fan/compressor[J]. Journal of Aerospace Power, 2014, 29(4): 917-926.

[6] 梁东, 王利, 弓志强, 等. 一种风扇/压气机声模态测 量分析方法[J]. 燃气涣轮试验与研究, 2015, 28(3): $49-53,62$. 
LANG Dong, WANG Li, GOMG Zhiqiang, et al.Research on acoustic mode measurement analysis method for a fan/compressor[J]. Gas Turbine Experiment and Research, 2015, 28(3): 49-53, 62.

[7] BERND H, JOERG R S. Causes of acoustic resonance in a high-Speed axial compressor[J]. Journal of Turbomachinery, 2008, 130(7): 1-9.

[8] CAMP T R. A study of acoustic response in a low speed multistage compressor[J]. Journal of Turbomachinery, 1999, 121: 36-43.

[9] 杨明绥, 刘思远, 王德友, 等. 航空发动机压气机声共 振现象初探[J]. 航空发动机, 2012, 38(5): 36-42.

YANG Mingsui, LIU Siyuan, WANG Deyou, et al. Study of acoustic resonance for aero engine compressors[J]. Aeroengine, 2012, 38(5): 36-42.

[10] 张波, 李健军. 基于 Hankel 矩阵与奇异值分解(SVD) 的滤波方法以及在飞机颤振试验数据预处理中的应用 [J]. 振动与冲击, 2009, 28(2): 162-166.

ZHANG Bo, LI Jianjun. Denoising method based on hankelmatric and SVD and its application in flight flutter testing data preprocessing[J]. Journal of Vibration and Shock, 2009, 28(2): 162-166.

[11] 赵学智, 叶邦彦, 陈统坚. 奇异值差分谱理论及其在车
床主轴箱故障诊断中的应用 $[\mathrm{J}]$. 机械工程学报, 2010, 46(1): 100-108.

ZHAO Xuezhi, YE Bangyan, CHEN Tongjian.Difference spectrum theory of singular value and its application to the fault diagnois of headstock of lathe[J]. Journal of Mechanical Engineering, 2010，46(1): 100-108.

[12] 彭培英, 申永军, 李黎阳. 基于奇异值分解技术的离心 机故障诊断[J]. 动力学与控制学报, 2008, 6(2): 165-168. PENG Peiying, SHEN Yongjun, LI Liyang.Centrifuge fault diagnosis based on singular value decomposition[J]. Journal of Dynamics and Control, 2008, 6(2): 165-168.

作者简介: 程礼(通信作者), 男, 1963 年出生, 博士, 教授, 博士研究 生导师。主要研究方向为航空发动机结构、强度、振动与故障诊断。

E-mail:846141917@qq.com

杨武奎, 男, 1975 年出生, 博士研究生。主要研究方向为航空发动机结 构、强度与振动。

E-mail: yangwukui1975@163.com

梁涛, 男, 1993 年出生, 硕士研究生。主要研究方向为航空发动机结构、 强度与振动。

E-mail: 1120094801@qq.com

文璧, 男, 1983 年出生, 硕士, 高级工程师。主要研究方向为航空发动 机特种测试技术, 故障诊断测试技术。

E-mail: 85282053@qq.com

姚东野, 男, 1977 年出生, 硕士, 讲师。主要研究方向为航空发动机结 构、强度与振动。

E-mail: 349795572@qq.com 